\title{
Gut microbiota and bronchopulmonary dysplasia
}

\author{
Kun Yang ${ }^{1}$, Shasha $\mathrm{He}^{1}$, and WENBIN DONG ${ }^{1}$ \\ ${ }^{1}$ The Affiliated Hospital of Southwest Medical University
}

March 19, 2021

\begin{abstract}
Bronchopulmonary dysplasia is a relatively common and severe complication of prematurity, and its pathogenesis remains ambiguous. Revolutionary advances in microbiological analysis techniques, together with the growing sophistication of the gut-lung axis hypothesis, have resulted in more studies linking gut microbiota dysbiosis to the occurrence and development of bronchopulmonary dysplasia. The present article builds on current findings to examine the intrinsic associations between gut microbiota and bronchopulmonary dysplasia. The gut microbiota affects bronchopulmonary dysplasia via several potential mechanisms including alteration of the gut-lung axis, promotion of inflammation and the ensuing growth effects, therefore these are also investigated. By evaluating the potential mechanisms, new therapeutic targets and potential therapeutic modalities for BPD can be identified from a microecological perspective.
\end{abstract}

\section{INTRODUCTION}

In recent decades, the survivorship of preterm infants has increased significantly thanks to the improved lifesaving techniques and precise application of medication, but the concomitant challenge is that the incidence of bronchopulmonary dysplasia (BPD) is gradually rising ${ }^{1}$. BPD is a severe medical challenge and a tremendous trial for frail preterm infants, in part because the short- and long-term respiratory and neurological complications associated with BPD severely affect the quality of life of preterm infants ${ }^{2}$. Furthermore, no unified consensus has so far been reached regarding the definition, pathology, pathogenesis and treatment options of BPD. Therefore, it has become imperative to understand this condition and dissect BPD from different angles. Recent evidence has demonstrated the critical contribution of the gut microbiota, as the most prominent component of the micro-ecological environment, in several respiratory diseases ${ }^{3}$. Notably, a growing body of research has examined BPD and gut microbiota dysbiosis associations. Despite this, few comprehensive reports have been published in this area. As such, this review's significance is to highlight the bidirectional impact of gut microbiota dysbiosis and BPD and establish the possible mechanisms by which gut microbiota dysbiosis affects BPD.

\section{A BRIEF RETROSPECT OF THE GUT MICROBIOTA}

The human micro-ecological environment is an incredibly intricate "super bacterial body" that coexists in organs such as the skin, lungs, and intestines, with specific bacterial colony patterns in different regions. The gut microbiota includes all the bacteria, archaea, viruses, eukaryotes, protozoans, and surroundings in the gastrointestinal tract ${ }^{4}$. About 100 trillion microbes live in the gastrointestinal tract, and the total number of cells and genomes of these bacteria are 10 times and more than 100 times greater respectively compared to humans ${ }^{5}$. Surprisingly, such massive colonization still maintains a long-term, mutually beneficial and deep relationship with the host.

The importance of the gut microbiota to the host lies, on the one hand, in the fact that it strengthens the intestinal epithelial mucosal barrier and is involved in the digestion of food, absorption of nutrients, metabolism of drugs, and defense against pathogens and toxins ${ }^{6,7}$. It also motivates intestinal ripening by releasing mucus, promoting angiogenesis, thickening the villi, widening the mucosal surface, and supporting 
cell proliferation ${ }^{8}$. Besides these actions, gut microbiota produces a variety of vital substances such as vitamins B12, folate, biotin, pyridoxine and thiamin, as well as metabolites including short-chain fatty acids (SCFA), bacteriocins, and microbial amino acids, which are involved in a variety of biological metabolic activities $^{6,7}$. Furthermore, the gut microbiota promotes growth by inducing insulin-like growth factor-1 signaling ${ }^{9}$.

On the other hand, the gut microbiota activates and regulates a range of immune cells such as innate hematolymphoid cells, natural killer cells, and helper lymphocytes, reflecting its essential contribution in driving the host immune function ${ }^{10}$. SCFA, polysaccharide A, $\alpha$-galactosylceramide, and tryptophan metabolites from the gut microbiota activate interleukin-22, interleukin-17, IgA, and Reg3r which participate in immune regulation ${ }^{11}$. SCFA also stimulates the production of the anti-inflammatory factors interleukin-10, interleukin-21; murein lipoprotein, a cell wall component of gut microbiota, and can also promote IgG release $^{12}$. Conceivably, healthy colonization of gut microbiota during infancy has profound implications for future immunity and metabolism ${ }^{13,14}$.

Notably, the gut microbiota's significance probably dictates its rapid establishment shortly after birth but may be influenced by prenatal non-sterile intrauterine conditions, a special placental microbiota ${ }^{15}$. In the early stages of life,Proteobacteria and Actinobacteria are significant members of the gut microbiota; over time, the diversity and abundance of the intestinal microbiota evolved until early childhood acquired a microbial composition similar to that of adults ${ }^{16}$. By this stage the microbiota consists mainly of Proteobacteria ,Firmicutes ,Actinobacteria, Verrucomicrobia, andBacteroidetes at the phylum-level, with Firmicutes and Bacteroidetes accounting for 90 percent of the total present ${ }^{4,6}$. Other studies have shown that Gram-positive cocci, Enterobacteriaceae orBifidobacteriaceae are the major components of infant gut microbiota, which gradually transition to a predominance ofBifidobacteriaceae ${ }^{17}$. In contrast, the gut microbiota of preterm infants in the neonatal intensive care unit (NICU) were more likely to show a sequential switch from Bacillito Gammaproteobacteria to Clostridia ${ }^{18,19}$. Overall, this dynamic transition in the gut microbiota is likely an adaptive alteration undertaken by the evolving neonatal population. However, this adds to the challenge of understanding the gut microbiota during the neonatal period. Therefore, it is not surprising that some studies have reported differing or even opposing results in gut microbiota composition during the neonatal period.

It is noteworthy that throughout gut microbiota development, its composition is extremely susceptible to a variety of elements as shown in Figure $\mathbf{1}^{15,16}$. Antibiotics are presumed to be one of the most important and sensitive factors in causing insult to the gut microbiota. For example, early administration of oral antibiotics to newborn rats resulted in significant gut microbiota changes, indicating that Proteobacteria and Bacteroidetesreplaced Firmicutes and Actinobacteria, and the concomitant descent in the proportion of Clostridia and Bacilli was accompanied by an increment in GammaProteobacteria ${ }^{20}$. Furthermore, remarkable changes in gut microbiota diversity were observed in infants exposed to antibiotics both prenatally and during the intrapartum stages, especially in terms of decreases observed in Bacteriodetes and Bifidobacteria and an increase inProteobacteria ${ }^{21}$. Another study showed that postnatal antibiotic exposure was associated with significantly lower levels of Enterococcus and Lactobacillus in the intestines of preterm infants, with Enterococcus thought to be associated with immunomodulation ${ }^{22}$ and Lactobacillusexhibiting powerful anti-inflammatory properties ${ }^{23}$.

Previous studies have shown that early and prolonged antibiotic exposure increases BPD risk in deficient birth weight infants ${ }^{24}$. One possible explanation is that antibiotics lead to alterations in the taxonomy and functional diversity of the gut microbiota, prolonging the time to restore healthy colonization and increasing foreign pathogen invasion opportunities ${ }^{25}$. Antibiotics can also whittle down the concentrations of SCFA ${ }^{20}$, which are considered to have antibacterial and anti-inflammatory properties ${ }^{26}$. Besides this, antibiotics diminish the abundance ofLactobacillus, which may delay host weight gain ${ }^{27}$ and further affect $\mathrm{BPD}^{28}$.

Gestational age is another factor that affects the gut microbiota. Premature infants showed reduced microbiota diversity compared to full-term infants, with decreased amounts of Bifidobacterium andBacteroides and increased amounts of Enterococcus andProteobacteria in those born early ${ }^{4}$. Other research has found 
a significantly increased abundance ofStaphylococcaceae in NICU preterm infants, accompanied by delayed transition to Bifidobacteriaceae; a decrease inBifidobacteriaceae possibly results in acetate concentration and $\mathrm{pH}$ changes, which are also associated with premature health ${ }^{17}$. In part, preterm infants are usually transported to environmentally stringent NICU, thus limiting their contact with mothers and the surrounding environment, which results in a delayed or impaired conversion of the gut microbiota from facultative anaerobic to completely anaerobic bacteria ${ }^{14}$. This may result in an insufficient abundance of Bifidobacterium andBacteroides and lead to increased pathogenic bacteria invasion.

Taken together, the gut microbiota probably exerts a tremendous impact on health. Maintaining gut microbiota diversity and stability potentially enhances host-specific resistance to the environment, as abundant species can mutually compensate for functional deficiencies ${ }^{29}$. Conversely, once the indicators representing the relative steady state of the gut microbiota, including resistance, resilience, and functional redundancy, change dramatically, the gut microbiota may have a diminished or delayed ability to recover its original phenotype $^{13}$. Once the original phenotype cannot be regained, gut microbiota dysbiosis can occur, which may result in onset of several gastrointestinal and extraintestinal diseases, as shown in Figure 1 .

\section{ASSOCIATION OF GUT MICROBIOTA WITH BPD}

Considerable numbers of studies have demonstrated lung microbiota imbalances in patients with BPD ${ }^{30,31}$. This is of interest given that the intestinal and respiratory epithelium have many similarities, both anatomically and functionally ${ }^{32}$. Several studies have investigated gut microbiota dysbiosis in BPD patients. Research found that the relative abundance ofEscherichia and Shigella increased significantly, whileKlebsiella and Salmonella declined markedly in gut microbiota from infants born transvaginally with BPD compared to those without $\mathrm{BPD}^{33}$. Furthermore, the gut microbiota of preterm infants receiving mechanical ventilation was significantly enriched in Proteobacteria with age, whilst Firmicutesnumbers declined sharply and Staphylococcus was the dominant genus at the genus level ${ }^{34}$. Another case-controlled study showed that the operational taxonomic units, relative abundance, and Shannon index of the gut microbiota were significantly reduced in BPD infants 28 days after birth ${ }^{35}$. Additionally, BPD severity probably correlated positively with the risk of gut anaerobic microenvironment disruption ${ }^{35}$.

Interestingly, it also appears that changes in the gut microbiota of BPD can be viewed from the perspective of metabonomics. Pintus et al. ${ }^{36}$ collected urine samples from 18 newborns seven days after birth and identified that the BPD and non-BPD groups displayed distinct metabolic patterns. Specifically, alanine and betaine increased, while trimethylamine-N-oxide (TMAO), lactate, and glycine decreased in the BPD group. Since the gut microbiota mediates the formation and production of TMAO to some extent ${ }^{37}$, it can be assumed that the decreased levels of TMAO in BPD patients reflect alterations in their gut microbiota.

These data directly or indirectly reveal the fact that gut microbiota dysbiosis occurs in BPD infants. In fact, gut microbiota dysregulation in turn probably also affects the progression of BPD. For instance, in the BPD model of perinatal maternal antibiotic exposure (MAE), the destruction of the gut microbiota diminished pulmonary vascular density, thickened the alveolar septum under hyperoxia, induced alveolar simplification and promoted more severe BPD characterized by pulmonary fibrosis ${ }^{38}$. It is worth mentioning that MAE was sufficient to induce pulmonary vascular obstacle even under normoxia conditions, suggesting that lung structural abnormalities are associated with microbiota dysbiosis ${ }^{38}$. Another study also showed that MAE remarkably diminished the abundance of commensal bacteria in the mouse gut, which aggravated hyperoxiainduced impairment of alveolar and angiogenesis ${ }^{39}$. Moreover, a cohort study in people showed a markedly increased risk of death or BPD in very low birth weight (VLBW) infants who received antibiotics two weeks after birth ${ }^{40}$. The increased risk of death or BPD was positively correlated with antibiotic exposure duration, meaning that each additional day of antibiotics was associated with an approximately $13 \%$ increased BPD risk $^{40}$.

Paradoxically, however, Althouse et al. ${ }^{41}$ reported that although MAE caused gut microbiota dysbiosis, this did not dramatically exacerbate the hyperoxia lung injury phenotype. Another study showed that the lung microbiota is more likely to influence BPD severity than the gut microbiota ${ }^{42}$. One possibility is that 
in addition to BPD, there are likely multiple other factors influencing gut microbiota, this increases the uncertainty of both associations, suggesting that the correlation between gut microbiota and BPD needs further investigation.

Hyperoxia is known to be a high-risk factor for BPD. One possible reason is that hyperoxia alters the gut microbiota, leading to the pathogen invasion and inflammation involved in BPD development. For example, hyperoxia exposure dramatically elevated amounts ofEnterobacteriaceae ${ }^{43}$, Proteobacteria and Epsilonbacteraeota ${ }^{44}$ in mouse intestines. Another murine intestinal tract showed that hyperoxia reversed the antibiotic-induced decreases in Bacteroidales andAlistipes and incerases of Akkermansia ${ }^{41}$. The gut of rats exposed to hyperoxia conditions also showed enrichment of Streptococcus and Gammaproteobacteria and Proteusdeficiencies ${ }^{45}$. Furthermore, Ashley et al. ${ }^{46}$ showed that following 72 hours of hyperoxia exposure, the intestinal tracts of mice exhibited pronounced decreases in the Firmicuts and Ruminococcaceae families and significantly increased Bacteroidetes were present; this gut microbiota dysbiosis possibly correlated with the degree of lung inflammation. Notably, the authors demonstrated that dysregulation of the lung and gut microbiota occurred prior to lung injury, suggesting that the microbiota dysbiosis contributed to the formation of hyperoxia-induced lung injury in mice ${ }^{46}$. In contrast, hyperoxia-exposed germ-free mice showed diminished structural and functional lung damage and had attenuated inflammatory infiltration compared to non-germ-free mice, suggesting a role for the microbiota in the development of $\mathrm{BPD}^{47}$.

Another aspect to consider is that hyperoxia disrupts intestinal epithelial cells, causing changes to the secretory component proteins, thus affecting mucosal immunity ${ }^{48}$. The intestine of hyperoxia-exposed rats showed significantly increased diamine oxidase, intestinal fatty acid binding protein, liver-type fatty acid binding protein, and concomitant decreased tight junction protein, suggesting impairment of the intestinal mucosal barrier ${ }^{49}$. Besides these differences, hyperoxia markedly elevated intestinal permeability and upregulated inflammatory markers such as toll-like receptor 4 (TLR4), nuclear factor kappa-B, tumor necrosis factor- $\alpha(\mathrm{TNF}-\alpha)$, interleukin-10, and interferon- $\gamma^{49,50}$. These results presumably facilitated the translocation of intestinal bacteria into the lungs, leading to an increase in pulmonary cytokines which affected lung health $^{51}$.

Metabolically, important considerations include fecal volatile organic compounds (VOCs), which are potentially intimately linked to BPD. VOCs probably affect lung function by altering the gas-liquid interface properties of pulmonary surfactant ${ }^{52}$, stimulating the production of pro-inflammatory factors ${ }^{53}$, and exacerbating oxidative stress ${ }^{54}$. Furthermore, VOCs alter the microRNA profile of lung tissue, which in turn impairs lung health ${ }^{55}$. Research has identified that VOCs can potentially predict the risk of lung injury in swine exposed to hyperoxia ${ }^{56}$. Similarly, VOCs can help diagnose and predict the onset of acute respiratory distress syndrome ${ }^{57}$ and $\mathrm{BPD}^{58}$ early. One study revealed that several fecal VOCs, such as tetradecane, Nnitrosopyrrolidine, and trichloretene, were significantly elevated in BPD patients ${ }^{59}$. Other studies confirmed that changes in fecal VOCs were intimately related to BPD severity ${ }^{60}$.

Notably, fecal VOC analysis is considered to be an accurate diagnostic tool for gastrointestinal diseases, and transformations in the gut microbiota play an etiological role. In other words, fecal VOCs largely reflect the composition, function, and interaction of gut microbiota ${ }^{61}$. Thus, from this perspective, gut microbiota dysbiosis likely causes changes in fecal VOCs, which further affects BPD.

Briefly, gut microbiota and BPD presumably mutually influence each other, but extensive experiments are needed to elucidate the specific mechanisms regarding their bidirectional effects. It is also necessary to establish the intrinsic link between gut microbiota dysbiosis and BPD through more direct experiments, rather than relying on antibiotic exposure, as antibiotics can damage microbiota in other parts of the host. Furthermore, it remains to be fully understood whether other gut microbiota members such as archaea, viruses, eukaryotes, and protozoans influence BPD.

\section{POSSIBLE MECHANISMS OF GUT MICROBIOTA INFLUENCING BPD}

Although the causal relationship between BPD and gut microbiota and their exact interaction mechanisms are not sufficiently well defined, a growing body of research suggests that gut microbiota dysbiosis can impair 
the gut-lung axis, activate inflammation, and influence growth; as shown in Figure 2 , these outcomes potentially compromise BPD further.

\section{Gut microbiotadysbiosis alters the gut-lung axis}

The gut microbiota may influence BPD by mediating lung immunity. A meaningful way to accomplish this process is the gut-lung axis, which can be articulated as a dysbiosis of the gut/lung microbiota that affects lung/intestinal health ${ }^{26}$. Achieving this sophisticated bidirectional crosstalk may involve both the circulatory and lymphatic immune systems. On the one hand, intestinally produced bacterial products (e.g., cellular debris, metabolites) reach the pulmonary circulation via the systemic circulation, stimulating lung immune cells (e.g., dendritic cells (DCs), macrophages, T cells, and neutrophils) to activate inflammation and trigger lung injury ${ }^{62}$. On the other hand, intestinal DCs recognize the antigens and migrate towards the mesenteric lymph nodes or gut-associated lymphoid tissue in order to activate B and T cells, which consequently reach the lung epithelium and activate the lung immune response ${ }^{62}$.

For example, the gut microbiota can upregulate interleukin-17A levels which initiate granulocyte-macrophage colony-stimulating factor signaling, which further enhances the abilities of alveolar macrophages in relation to scavenging Streptococcus pneumoniae and Klebsiella pneumoniae ${ }^{63}$. Neonatal mice exposed to gut commensal bacteria had better lung mucosal defenses than those not exposed, presumably due to intestinal DCs inducing CCR4 expression which promoted interleukin-22-producing group 3 innate lymphoid cell migration into the lungs, thereby enhancing lung immunity; importantly, CD $103^{+} \mathrm{CD}_{11} \mathrm{~b}^{+} \mathrm{DCs}$ recognition of the gut microbiota is required to initiate this process ${ }^{64}$. Furthermore, the high mortality of pulmonary viral infections in antibiotic-exposed mice may be due to immune compromise caused by dysbiosis of the gut microbiota, leading to an increase in lung interferon- $\gamma$, interleukin- 6 , and CCL2 and a decrease in Treg cells ${ }^{65}$.

These data suggest that the gut-lung axis links the bidirectional crosstalk between the intestine and lung. Microbiota dysbiosis in either side of the gut and lung is likely to have potentially dramatic impacts on the other side via the gut-lung axis. Given that previous studies have examined the association between dysbiosis of the lung microbiota with the occurrence and development of $\mathrm{BPD}^{66,67}$, we have constructed a lung microbiota-gut microbiota-BPD triangle, as shown in Figure 3. We propose that each factor in the triangle is probably strongly interrelated. Hence, it is not difficult to infer that maintaining the stability of the host's entire microbiota has profound implications for preventing or improving BPD.

Although the function of the gut-lung axis in other respiratory diseases has been well-defined, the specific mechanisms of how the gut microbiota affects the lung via the gut-lung axis in BPD have been investigated less. Future directions exploring more experimental data in relation to the gut-lung axis in BPD may be required to further explain this complex area. Fully grasping this relationship is imperative as new potential therapeutic targets for BPD may be elucidated.

\section{Gut microbiota dysbiosis promotes inflammation}

An essential aspect in the development of BPD is inflammation activation. Recruitment of neutrophils and macrophages to preterm immature lungs in response to chemokines contributes to the production of several inflammatory factors such as interleukin-1, interleukin-6, interleukin- 8 , TNF- $\alpha$ and transforming growth factor- $\beta$ (TGF- $\beta$ ), resulting in lung injury ${ }^{68}$, an essential pathological process in BPD. In addition, recent studies have shown that the decrease of interleukin- $17 \mathrm{~A}^{69}$, as a microbial signal-related cytokine ${ }^{38}$, and anti-inflammatory factor interleukin- $10^{70}$, is strongly associated with BPD.

Notably, the gut microbiota appears to exert an extraordinarily vital function in regulating lung inflammation. The gut microbiota drives the secretion of interleukin- $1 \beta$, interleukin- 6 , and TGF- $\beta^{71,72}$, and its dysregulation causes a decrease in both interleukin-17A and interleukin-22, while altering interleukin-6 and TNF- $\alpha$ signaling ${ }^{38}$. For example, gut microbiota dysbiosis caused significantly elevated levels of interleukin- 6 in bronchoalveolar lavage fluid from mice with lipopolysaccharide-induced lung inflammation ${ }^{73}$. Furthermore, findings suggest that the metabolic state of alveolar macrophages is significantly altered in response 
to gut microbiota dysbiosis ${ }^{74}$. In contrast, supplementation with acetate, generated by the gut microbiota, dramatically diminished the levels of inflammation in the lungs of neonatal mice exposed to hyperoxia ${ }^{75}$. Moreover, fecal microbiota transplantation attenuated inflammatory cell infiltration and interstitial lung exudates in acute lung injury in rats by downregulating the expression of TNF- $\alpha$, interleukin- $1 \beta$, interleukin- 6 , and TGF- $\beta 1^{76}$. Similarly, fecal microbiota transplantation has been shown to alleviate radiation-induced lung inflammation ${ }^{77}$. Additionally, mice with gut microbiota dysbiosis that received fecal microbiota transplantations showed earlier recovery of both TNF- $\alpha$ and interleukin-10 levels following Streptococcus pneumoniaeinfection ${ }^{74}$.

Furthermore, the gut microbiota probably affects BPD by influencing the expression of specific immune genes in the blood ${ }^{33}$. For example, MAE increases the number of CD45-positive cells and granulocytes, leading to over-immunity, resulting in lung injury ${ }^{38}$. Besides this, metabolites produced by the gut microbiota, such as lipopolysaccharide, recognized by TLR4 via the pathogen-associated molecule patterns pathway, caused an increase in interleukin- $1 \beta$, which further activated nuclear factor kappa-B and formed an inflammatory cascade leading to lung injury ${ }^{43}$.

Surprisingly, gut microbiota and its metabolites also seem to be associated with pulmonary fibrosis ${ }^{78}$. For example, TLR4 recognizes the damage-associated molecular pattern signal produced by the gut microbiota and activates myeloid differentiation 2/TLR4-dependent fibroblasts under the drive of myeloid differentiation 2 , resulting in lung fibrosis ${ }^{79}$.

Collectively, gut microbiota dysbiosis initiates inflammation partly via direct transfer of bacteria to the lungs ${ }^{80}$ and partly through the release of specific immune signals such as polysaccharide A, sphingolipids, which are taken up by immune cells triggering an inflammatory response. Moreover, gut microbiota dysregulation causes impaired intestinal epithelial integrity, allowing microorganisms and metabolites to directly enter the bloodstream, leading to systemic inflammation. When the developing lungs receive these abnormal or amplified inflammatory signals, they alter the lungs perception of their surroundings, leading to chronic inflammation ${ }^{32}$.

\section{Gut microbiota dysbiosis affects growth}

It is widely accepted that malnutrition is one of the most critical factors leading to the occurrence and deterioration of BPD. Previous studies have shown that adequate early energy and protein supply are significantly negatively correlated with BPD risk $^{28,81}$. For VLBW or extremely premature (EPT) infants, elimination of undernutrition as a means of recovery from BPD may be beneficial. Notably, there appears to be a strong correlation and partial overlap between postnatal growth restriction, gut microbiota dysbiosis, and BPD, especially between gut microbiota and preterm infant nutrition, although the causal relationship between the three still needs to be confirmed ${ }^{82}$.

Mice receiving gut microbiota from malnourished infants developed growth disturbances and metabolic abnormalities, whereas mice receiving gut microbiota from healthy infants did not. Interestingly the mice received the gut microbiota from undernourished infants gained in terms of growth advancement when the two groups of mice cohabited ${ }^{83}$. A microbiota-directed complementary food study showed improvements in the nutritional status of gnotobiotic animals and promoted growth, bone formation, neurodevelopment, and enhanced immune function were observed in malnourished children ${ }^{84}$. Furthermore, Lactobacillus plantarum helped maintain weight and length in germ-free mice exposed to chronic malnutrition by partially eliminating peripheral tissue resistance to growth hormone and insulin-like growth factor-1, thus illustrating the importance of the gut microbiota in promoting growth ${ }^{85}$.

Actinobacteria, Proteobacteria, and Firmicutes at the phylum level of gut microbiota were remarkably correlated with nutritional intake. Actinobacteria and Proteobacteriacorrelate with lipid intake, Firmicutes with protein, and all three are associated with carbohydrates, these presumably facilitate increased calories uptake and growth ${ }^{19}$. In addition, the gut microbiota is involved in energy metabolism as it regulates the levels of several organic acids such as pyruvate, citric acid, fumaric acid and malic acid, and is intrinsic to lipid metabolism as the microbiota regulates lipase activity ${ }^{12}$. For example, gut microbiota dysbiosis 
releases specific signals to accelerate lipolysis and fatty acid oxidation, which is probably a contributor to slow growth in malnourished infants ${ }^{86}$. Moreover, SCFA (butyrate, acetate and propionate) and other specific substances (trimethylamine, indolepropionic acid) secreted by the gut microbiota contribute crucially to various nutrient metabolic processes including food fermentation and transformation. Specifically, butyrate helps collect energy, propionate senses gluconeogenesis and satiety signals, acetate is involved in cholesterol and fat metabolism, gut microbial enzymes regulate bile acid metabolism, and indolepropionic acid fights free radicals ${ }^{29}$.

Recent studies indicated that growth disorders were strongly associated with reducing gut microbiota diversity and maturity.Staphylococcaceae and Enterobacteriaceae were the dominant bacteria observed during the growth decline phase, whereasStreptococcus and Bifidobacterium were present in relatively high proportions during the growth catch-up period ${ }^{86}$. An obvious consideration is the weakened ability of Enterobacteriaceae (a significant member within theGammaproteobacteria family) to decompose human milk oligosaccharides and engender less butyrate and vitamins, which cripples intestinal digestion and absorption ${ }^{87}$. Furthermore, Yee et al. ${ }^{88}$ showed a relationship between the growth of 83 VLBW infants and longitudinal gut microbiota changes. They discovered that weight gain was related to the alpha diversity of the microbiota, and length gain was related to the fluctuation amplitude of beta diversity and maturity. Gut microbiota dysbiosis impedes VLBW growth through multiple pathways, such as weakened or disrupted synthesis of polysaccharides and amino acids, consequently making it more susceptible to pathogens ${ }^{87}$.

It is of note that weight-gain at different age stages is possibly influenced by the composition of the gut microbiota at specific times. For example, the gut microbiota during early postnatal days impacts the weight of preterm infants at one month of age. In these cases the abundance of Staphylococcus and Enterococcus wasnegatively correlated with weight-gain, whereas Weissella was positively associated with weight-gain ${ }^{89}$. Similarly, the diversity and maturity of the gut microbiota at month 6 postnatally were correlated with weight-gain at 6-12 months of age, during which time Proteobacteria and Bacteroidetes were positively correlated with weight-gain, whereas Actinobacteria were negatively correlated with weight-gain ${ }^{90}$.

These data suggest that gut microbiota plays a critical role in growth. Gut microbiota dysbiosis and malnutrition are in turn involved in BPD potentially through mechanisms such as altering epigenetics, promoting inflammation and oxidative stress, changing intestinal permeability, modifying vascular and lymphatic vessel development, and by affecting micronutrients ${ }^{82}$.

\section{CONCLUSIONS}

BPD continues to be a challenge for preterm infants worldwide. Today, BPD has an increasing incidence rate, and whilst there are no definitive treatment options, several intricate mechanisms have been identified and associated with the condition. The gut microbiota, as an "invisible organ" of the host, has been shown to play critical roles in many diseases throughout all of the body systems. A growing number of studies have shown an intrinsic association between gut microbiota and BPD. Although the causal relationship between the two remains to be determined, gut microbial dysbiosis may affect BPD in a number of ways, including altering the gut-lung axis, promoting inflammation, and affecting growth. Therefore, fecal microbiota transplantation or probiotics potentially represent therapeutic options for the treatment or prevention of BPD. Despite these advances, investigations into the associations between gut microbiota and BPD currently remain in the preliminary exploratory stages, and substantial questions need to be addressed urgently. Therefore, further experiments are required to demonstrate the feasibility and safety of this therapeutic modality before identifying gut microbiota as a therapeutic target for BPD. Future directions also need to focus on refining the knowledge related to the specific mechanisms by which the gut microbiota affects BPD.

In conclusion, a comprehensive understanding of the relationship between the gut microbiota and BPD is necessary and urgent. Comprehending the relationships may add new dimensions to the pathogenesis of $\mathrm{BPD}$ and be used as targets for more promising therapeutic approaches.

\section{ACKNOWLEDGEMENTS}


The authors would like to express their gratitude to EditSprings (https://www.editsprings.com/) for the expert linguistic services provided.

\section{FUNDING}

This article was supported by the National Natural Science Foundation of China (81571480) and Luzhou Municipal People's Government-Southwest Medical University Science and Technology Strategic Cooperation Project (2020LZXNYDJ03)

\section{CONFLICTS OF INTEREST}

The authors have no conflicts of interest to declare.

\section{Abbreviations:}

BPD, bronchopulmonary dysplasia

SCFA, short-chain fatty acids

NICU, neonatal intensive care unit

TMAO, trimethylamine-N-oxide

MAE, maternal antibiotic exposure

VLBW, very low birth weight

VOCs, volatile organic compounds

DCs, dendritic cells

TNF- $\alpha$, tumor necrosis factor- $\alpha$

TGF- $\beta$, transforming growth factor- $\beta$

TLR4, toll-like receptor 4

EPT, extremely prematu

\section{References}

1. Thébaud B, Goss KN, Laughon M, et al. Bronchopulmonary dysplasia.Nat Rev Dis Primers. 2019;5(1):78.

2. Baker EK, Cheong JLY, Doyle LW. Short- and Long-Term Outcomes After Bronchopulmonary Dysplasia. In: Kallapur SG, Pryhuber GS, eds. Updates on Neonatal Chronic Lung Disease. Elsevier; 2020:291-305.

3. Chunxi L, Haiyue L, Yanxia L, Jianbing P, Jin S. The Gut Microbiota and Respiratory Diseases: New Evidence. J Immunol Res.2020;2020:2340670.

4. Illiano P, Brambilla R, Parolini C. The mutual interplay of gut microbiota, diet and human disease. FEBS J. 2020;287(5):833-855.

5. Baeckhed F, Ley RE, Sonnenburg JL, Peterson DA, Gordon JI. Host-Bacterial Mutualism in the Human Intestine. Science.2005;307(5717):1915-1920.

6. Thursby E, Juge N. Introduction to the human gut microbiota.Biochem J. 2017;474(11):1823-1836.

7. Sakkas H, Bozidis P, Touzios C, Kolios D, Gartzonika C. Nutritional Status and the Influence of the Vegan Diet on the Gut Microbiota and Human Health. Medicina (Kaunas, Lithuania). 2020;56(2):88.

8. Becattini S, Taur Y, Pamer EG. Antibiotic-Induced Changes in the Intestinal Microbiota and Disease. Trends in Molecular Medicine.2016:S1471491416300077.

9. Yan J, Charles JF. Gut Microbiota and IGF-1. Calcif Tissue Int. 2018;102(4):406-414. 
10. Belizário JE, Faintuch J, Garay-Malpartida M. Gut Microbiome Dysbiosis and Immunometabolism: New Frontiers for Treatment of Metabolic Diseases. Mediators of inflammation. 2018;2018:2037838.

11. McDermott AJ, Huffnagle GB. The microbiome and regulation of mucosal immunity. Immunology. 2014;142(1):24-31.

12. Shukla SD, Budden KF, Neal R, Hansbro PM. Microbiome effects on immunity, health and disease in the lung. Clin Transl Immunology.2017;6(3):e133.

13. Moya A, Ferrer M. Functional Redundancy-Induced Stability of Gut Microbiota Subjected to Disturbance. Trends in microbiology.2016:S0966842X16000263.

14. Nguyen TTB, Chung H-J, Kim H-J, Hong S-T. Establishment of an ideal gut microbiota to boost healthy growth of neonates. Critical reviews in microbiology. 2019;45(1):118-129.

15. Tirone C, Pezza L, Paladini A, et al. Gut and Lung Microbiota in Preterm Infants: Immunological Modulation and Implication in Neonatal Outcomes. Front Immunol. 2019;10:2910.

16. Rodrguez JM, Murphy K, Stanton C, et al. The composition of the gut microbiota throughout life, with an emphasis on early life. 2015.

17. Tauchi H, Yahagi K, Yamauchi T, et al. Gut microbiota development of preterm infants hospitalised in intensive care units. Benef Microbes. 2019;10(6):641-651.

18. La Rosa PS, Warner BB, Zhou Y, et al. Patterned progression of bacterial populations in the premature infant gut. Proc Natl Acad Sci USA. 2014;111(34):12522-12527.

19. Grier A, Qiu X, Bandyopadhyay S, et al. Impact of prematurity and nutrition on the developing gut microbiome and preterm infant growth. Microbiome. 2017;5(1):158.

20. Stanisavljević S, Čepić A, Bojić S, et al. Oral neonatal antibiotic treatment perturbs gut microbiota and aggravates central nervous system autoimmunity in Dark Agouti rats. Scientific Reports. 2019;9(1).

21. Dierikx TH, Visser DH, Benninga MA, et al. The influence of prenatal and intrapartum antibiotics on intestinal microbiota colonisation in infants: A systematic review. The Journal of infection.2020;81(2):190204.

22. Jia J, Xun P, Wang X, et al. Impact of Postnatal Antibiotics and Parenteral Nutrition on the Gut Microbiota in Preterm Infants During Early Life. JPEN Journal of parenteral and enteral nutrition.2020;44(4):639654.

23. Vareille-Delarbre M, Miquel S, Garcin S, et al. Immunomodulatory Effects of Lactobacillus plantarum on Inflammatory Response Induced by Klebsiella pneumoniae. Infect Immun. 2019;87(11):e00570-00519.

24. Novitsky A, Tuttle D, Locke RG, Saiman L, Mackley A, Paul DA. Prolonged early antibiotic use and bronchopulmonary dysplasia in very low birth weight infants. American journal of perinatology.2015;32(1):43-48.

25. Lange K, Buerger M, Stallmach A, Bruns T. Effects of Antibiotics on Gut Microbiota. Digestive diseases (Basel, Switzerland).2016;34(3):260-268.

26. Yang K, Dong W. Perspectives on Probiotics and Bronchopulmonary Dysplasia. Frontiers in pediatrics. 2020;8:570247.

27. Miao Z, Cheng R, Zhang Y, et al. Antibiotics can cause weight loss by impairing gut microbiota in mice and the potent benefits of lactobacilli. Bioscience, biotechnology, and biochemistry.2020;84(2):411-420.

28. Klevebro S, Westin V, Stoltz Sjostrom E, et al. Early energy and protein intakes and associations with growth, BPD, and ROP in extremely preterm infants. Clinical Nutrition. 2019;38(3):1289-1295.

29. Valdes AM, Walter J, Segal E, Spector TD. Role of the gut microbiota in nutrition and health. BMJ. 2018;361:k2179. 
30. Lohmann P, Luna RA, Hollister EB, et al. The airway microbiome of intubated premature infants: characteristics and changes that predict the development of bronchopulmonary dysplasia. Pediatric Research. 2014;76(3):294-301.

31. Lal CV, Travers C, Aghai ZH, et al. The Airway Microbiome at Birth.Scientific Reports. 2016;6:31023.

32. Budden KF, Gellatly SL, Wood DL, et al. Emerging pathogenic links between microbiota and the gut-lung axis. Nat Rev Microbiol.2017;15(1):55-63.

33. Ryan FJ, Drew DP, Douglas C, et al. Changes in the Composition of the Gut Microbiota and the Blood Transcriptome in Preterm Infants at Less than 29 Weeks Gestation Diagnosed with Bronchopulmonary Dysplasia.mSystems. 2019;4(5):e00484-00419.

34. Gallacher D, Mitchell E, Alber D, et al. Dissimilarity of the gut-lung axis and dysbiosis of the lower airways in ventilated preterm infants. European Respiratory Journal. 2020;55(5):1901909.

35. Chen S-M, Lin C-P, Jan M-S. Early Gut Microbiota Changes in Preterm Infants with Bronchopulmonary Dysplasia: A Pilot Case-Control Study.American journal of perinatology. 2020.

36. Pintus MC, Lussu M, Dessì A, et al. Urinary H-NMR Metabolomics in the First Week of Life Can Anticipate BPD Diagnosis. Oxid Med Cell Longev. 2018;2018:7620671.

37. Wang Z, Klipfell E, Bennett BJ, et al. Gut flora metabolism of phosphatidylcholine promotes cardiovascular disease. Nature.2011;472(7341):57-63.

38. Willis KA, Siefker DT, Aziz MM, et al. Perinatal maternal antibiotic exposure augments lung injury in offspring in experimental bronchopulmonary dysplasia. American Journal of Physiology-lung Cellular and Molecular Physiology. 2020;318(2).

39. Chen C-M, Yang Y-CSH, Chou H-C. Maternal antibiotic exposure disrupts microbiota and exacerbates hyperoxia-induced lung injury in neonatal mice. Pediatric research. 2021.

40. Cantey JB, Huffman LW, Subramanian A, Marshall AS, Mallett LH. Antibiotic Exposure and Risk for Death or Bronchopulmonary Dysplasia in Very Low Birth Weight Infants. J Pediatr. 2016;181:289-293.

41. Althouse MH, Stewart C, Jiang W, Moorthy B, Lingappan K. Impact of Early Life Antibiotic Exposure and Neonatal Hyperoxia on the Murine Microbiome and Lung Injury. Scientific reports. 2019;9(1):14992.

42. Lauer T, Behnke J, Oehmke F, et al. Bacterial Colonization within the First Six Weeks of Life and Pulmonary Outcome in Preterm Infants <1000 g. Journal of clinical medicine. 2020;9(7).

43. Wedgwood S, Gerard K, Halloran K, et al. Intestinal Dysbiosis and the Developing Lung: The Role of Toll-Like Receptor 4 in the Gut-Lung Axis. Front Immunol. 2020;11:357.

44. Lo Y-C, Chen K-Y, Chou H-C, Lin IH, Chen C-M. Neonatal hyperoxia induces gut dysbiosis and behavioral changes in adolescent mice. J Chin Med Assoc. 2021.

45. Xing Z, Li Y, Liu G, He Y, Tao Y, Chen M. Hyperoxia provokes gut dysbiosis in rats. Critical care (London, England).2020;24(1):517.

46. Ashley SL, Sjoding MW, Popova AP, et al. Lung and gut microbiota are altered by hyperoxia and contribute to oxygen-induced lung injury in mice. Science translational medicine. 2020;12(556).

47. Dolma K, Freeman AE, Rezonzew G, et al. Effects of hyperoxia on alveolar and pulmonary vascular development in germ-free mice.American journal of physiology Lung cellular and molecular physiology. 2020;318(2):L421-L428.

48. Zhao M, Tang S, Xin J, Liu D. Influence of reactive oxygen species on secretory component in the intestinal epithelium during hyperoxia.Exp Ther Med. 2017;14(5):4033-4040. 
49. Liu DY, Lou WJ, Zhang DY, Sun SY. ROS Plays a Role in the Neonatal Rat Intestinal Barrier Damages Induced by Hyperoxia. Biomed Res Int. 2020;2020:8819195.

50. Chou H-C, Chen C-M. Neonatal hyperoxia disrupts the intestinal barrier and impairs intestinal function in rats. Exp Mol Pathol.2017;102(3):415-421.

51. Chen C-M, Chou H-C, Yang Y-CSH, Su EC-Y, Liu Y-R. Predicting Hyperoxia-Induced Lung Injury from Associated Intestinal and Lung Dysbiosis in Neonatal Mice. Neonatology. 2021:106-116.

52. Zhao Q, Li Y, Chai X, et al. Interaction of inhalable volatile organic compounds and pulmonary surfactant: Potential hazards of VOCs exposure to lung. J Hazard Mater. 2019;369:512-520.

53. Fischäder G, Röder-Stolinski C, Wichmann G, Nieber K, Lehmann I. Release of MCP-1 and IL-8 from lung epithelial cells exposed to volatile organic compounds. Toxicol In Vitro. 2008;22(2):359-366.

54. Yoon HI, Hong YC, Cho SH, et al. Exposure to volatile organic compounds and loss of pulmonary function in the elderly. The European respiratory journal. 2010;36(6):1270-1276.

55. Wang F, Li C, Liu W, Jin Y. Modulation of microRNA expression by volatile organic compounds in mouse lung. Environ Toxicol.2014;29(6):679-689.

56. Cronin WA, Forbes AS, Wagner KL, et al. Exhaled Volatile Organic Compounds Precedes Pulmonary Injury in a Swine Pulmonary Oxygen Toxicity Model. Front Physiol. 2019;10:1297.

57. Bos LDJ. Diagnosis of acute respiratory distress syndrome by exhaled breath analysis. Ann Transl Med. 2018;6(2):33.

58. Wright H, Bannaga AS, Iriarte R, Mahmoud M, Arasaradnam RP. Utility of volatile organic compounds as a diagnostic tool in preterm infants. Pediatric research. 2021;89(2):263-268.

59. Syzdykova M, Morenko M, Gatauova M, Temirkhnova R, Shnaider K. Role of Fecal Volatile Organic Compounds in the Diagnosis of Bronchopulmonary Dysplasia. Georgian Med News. 2020(308):80-84.

60. Berkhout DJC, Niemarkt HJ, Benninga MA, et al. Development of severe bronchopulmonary dysplasia is associated with alterations in fecal volatile organic compounds. Pediatric Research.2018;83(2):412-419.

61. El Manouni El Hassani S, Niemarkt HJ, Said H, et al. Fecal Volatile Organic Compounds in Preterm Infants Are Influenced by Enteral Feeding Composition. Sensors (Basel). 2018;18(9):3037.

62. Tan J-Y, Tang Y-C, Huang J. Gut Microbiota and Lung Injury.Advances in experimental medicine and biology. 2020;1238:55-72.

63. Brown RL, Sequeira RP, Clarke TB. The microbiota protects against respiratory infection via GM-CSF signaling. Nature communications. 2017;8(1):1512.

64. Gray J, Oehrle K, Worthen G, Alenghat T, Whitsett J, Deshmukh H. Intestinal commensal bacteria mediate lung mucosal immunity and promote resistance of newborn mice to infection. Science Translational Medicine. 2017;9(376):eaaf9412.

65. Grayson MH, Camarda LE, Hussain S-RA, et al. Intestinal Microbiota Disruption Reduces Regulatory $\mathrm{T}$ Cells and Increases Respiratory Viral Infection Mortality Through Increased IFN $\gamma$ Production. Front Immunol. 2018;9:1587.

66. Lal CV, Kandasamy J, Dolma K, et al. Early airway microbial metagenomic and metabolomic signatures are associated with development of severe bronchopulmonary dysplasia. Am J Physiol Lung Cell Mol Physiol. 2018;315(5):L810-1815.

67. Pammi M, Lal CV, Wagner BD, et al. Airway Microbiome and Development of Bronchopulmonary Dysplasia in Preterm Infants: A Systematic Review.The Journal of Pediatrics. 2019;204:126-133 e122. 
68. Speer CP. Inflammation and bronchopulmonary dysplasia. Paper presented at: Seminars in Neonatology 2003.

69. Witkowski SM, de Castro EM, Nagashima S, et al. Analysis of interleukins 6, 8, 10 and 17 in the lungs of premature neonates with bronchopulmonary dysplasia. Cytokine. 2020;131:155118.

70. Mao X, Qiu J, Zhao L, et al. Vitamin D and IL-10 Deficiency in Preterm Neonates With Bronchopulmonary Dysplasia. Frontiers in pediatrics. 2018;6:246.

71. Rosser EC, Oleinika K, Tonon S, et al. Regulatory B cells are induced by gut microbiota-driven interleukin-1 $\beta$ and interleukin-6 production. Nat Med. 2014;20(11):1334-1339.

72. Weiss GA, Hennet T. Mechanisms and consequences of intestinal dysbiosis. Cell Mol Life Sci. 2017;74(16):2959-2977.

73. Jacobs MC, Lankelma JM, Wolff NS, et al. Effect of antibiotic gut microbiota disruption on LPS-induced acute lung inflammation. PLoS ONE. 2020;15(11):e0241748.

74. Schuijt TJ, Lankelma JM, Scicluna BP, et al. The gut microbiota plays a protective role in the host defence against pneumococcal pneumonia. Gut. 2016;65(4):575-583.

75. Zhang Q, Ran X, He Y, Ai Q, Shi Y. Acetate Downregulates the Activation of NLRP3 Inflammasomes and Attenuates Lung Injury in Neonatal Mice With Bronchopulmonary Dysplasia. Frontiers in pediatrics.2020;8:595157.

76. Li B, Yin G-F, Wang Y-L, Tan Y-M, Huang C-L, Fan X-M. Impact of fecal microbiota transplantation on TGF- $\beta 1 /$ Smads/ERK signaling pathway of endotoxic acute lung injury in rats. 3 Biotech. 2020;10(2):52.

77. Nie X, Li L, Yi M, et al. The Intestinal Microbiota Plays as a Protective Regulator Against Radiation Pneumonitis. Radiat Res.2020;194(1):52-60.

78. Gong G-C, Song S-R, Su J. Pulmonary fibrosis alters gut microbiota and associated metabolites in mice: An integrated 16S and metabolomics analysis. Life sciences. 2020:118616.

79. Bhattacharyya S, Wang W, Qin W, et al. TLR4-dependent fibroblast activation drives persistent organ fibrosis in skin and lung. JCI Insight. 2018;3(13).

80. Dickson RP, Singer BH, Newstead MW, et al. Enrichment of the lung microbiome with gut bacteria in sepsis and the acute respiratory distress syndrome. Nat Microbiol. 2016;1(10):16113.

81. Uberos J, Jimenez-Montilla S, Molina-Oya M, Garcia-Serrano JL. Early energy restriction in premature infants and bronchopulmonary dysplasia: a cohort study. Br J Nutr. 2020:1-8.

82. Underwood MA, Lakshminrusimha S, Steinhorn RH, Wedgwood S. Malnutrition, poor post-natal growth, intestinal dysbiosis and the developing lung. J Perinatol. 2020.

83. Blanton LV, Charbonneau MR, Salih T, et al. Gut bacteria that prevent growth impairments transmitted by microbiota from malnourished children. Science. 2016;351(6275).

84. Gehrig JL, Venkatesh S, Chang H-W, et al. Effects of microbiota-directed foods in gnotobiotic animals and undernourished children. Science (New York, NY). 2019;365(6449).

85. Schwarzer M, Makki K, Storelli G, et al. Lactobacillus plantarum strain maintains growth of infant mice during chronic undernutrition.Science (New York, NY). 2016;351(6275):854-857.

86. Younge NE, Newgard CB, Cotten CM, et al. Disrupted Maturation of the Microbiota and Metabolome among Extremely Preterm Infants with Postnatal Growth Failure. Sci Rep. 2019;9(1):8167.

87. Groer M, Miller EM, Sarkar A, et al. Predicted Metabolic Pathway Distributions in Stool Bacteria in Very-Low-Birth-Weight Infants: Potential Relationships with NICU Faltered Growth. Nutrients.2020;12(5). 
88. Yee AL, Miller E, Dishaw LJ, et al. Longitudinal Microbiome Composition and Stability Correlate with Increased Weight and Length of Very-Low-Birth-Weight Infants. mSystems. 2019;4(1).

89. Arboleya S, Martinez-Camblor P, Solís G, et al. Intestinal Microbiota and Weight-Gain in Preterm Neonates. Front Microbiol.2017;8:183.

90. Kamng'ona AW, Young R, Arnold CD, et al. The association of gut microbiota characteristics in Malawian infants with growth and inflammation. Scientific reports. 2019;9(1):12893.

Figure 1 Factors affecting gut microbiota and dysbiosis of gut microbiota impact certain diseases

Figure 2 Possible mechanisms of gut microbiota influencing BPD

Figure 3 Lung microbiota-gut microbiota-BPD triangle
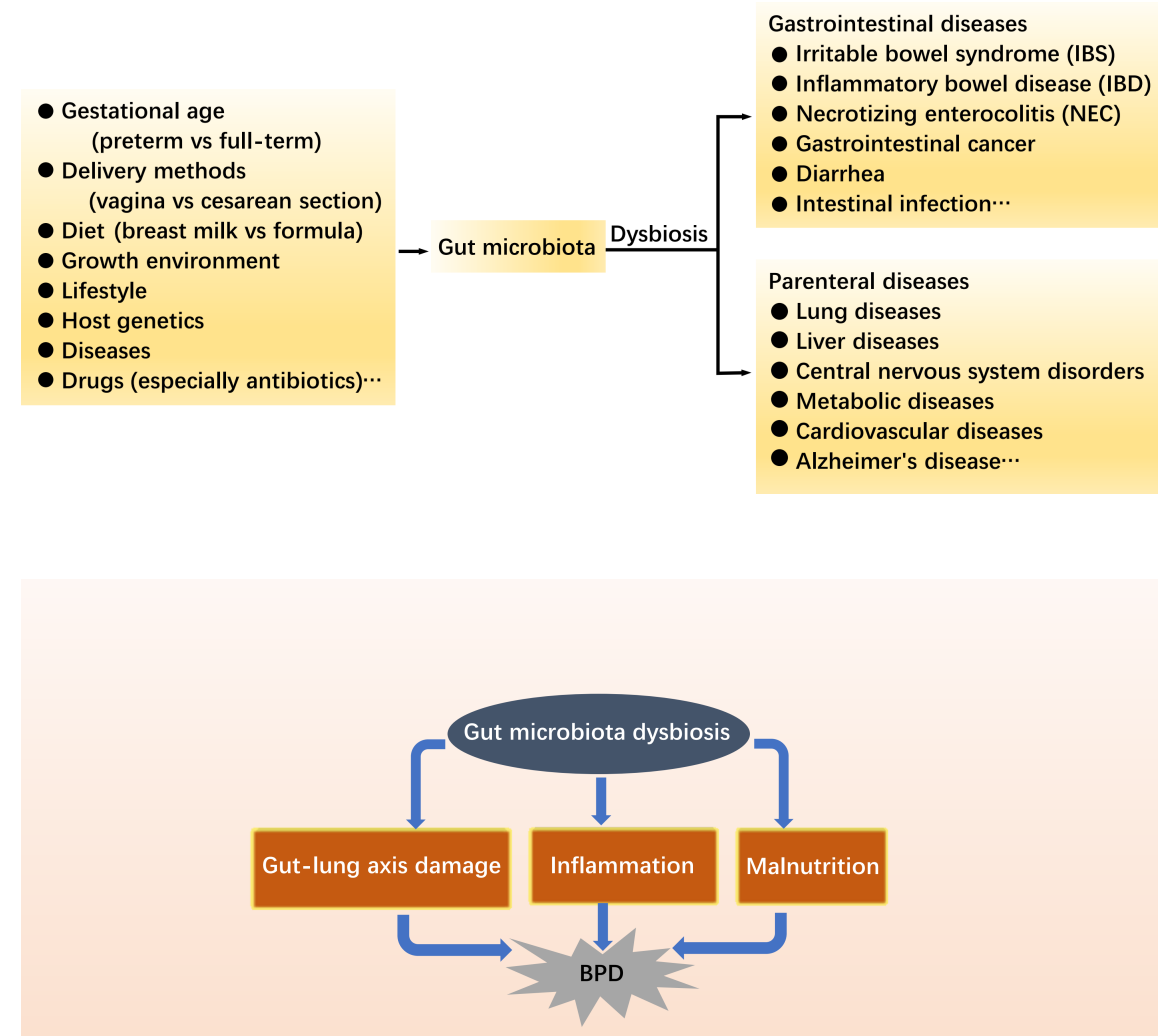


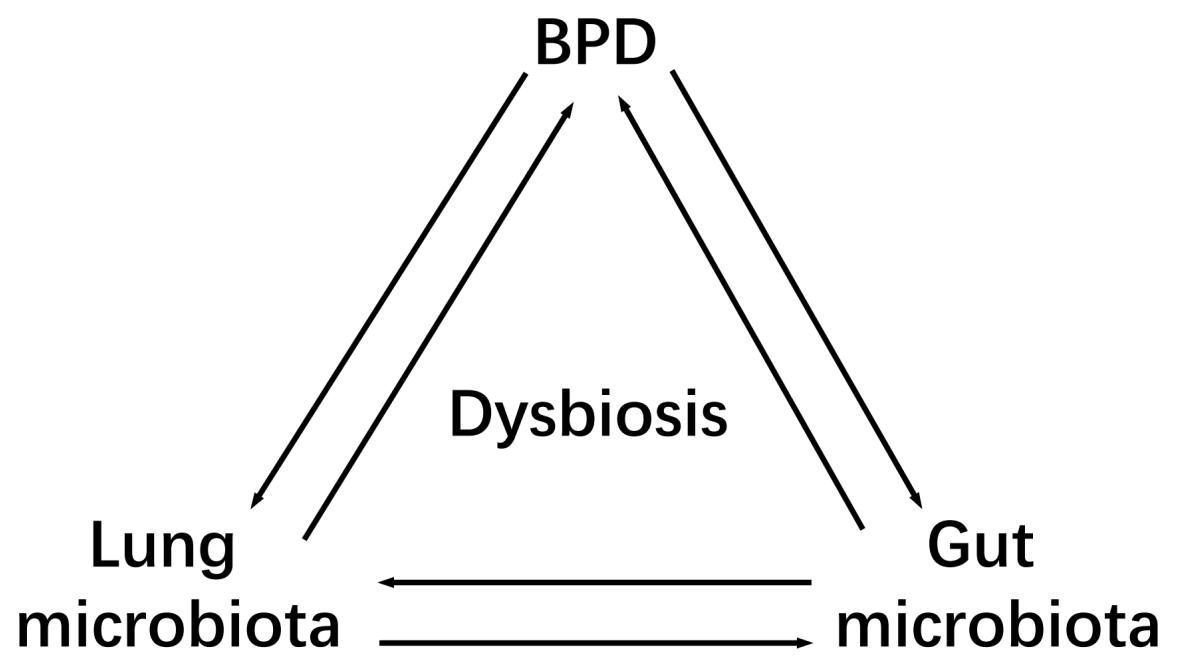

